\title{
QUANTUM SECTIONS AND GAUGE ALGEBRAS
}

\author{
L. LE BRUYN* and F. VAN OYSTAEyen
}

To the memory of P'ere Menal

\begin{abstract}
Using quantum sections of filtercd rings and the associated Rees rings one can lift the scheme structure on Proj of the associated graded ring to the Proj of the Rees ring. The algebras of interest here are positively filtered rings having a non-commutative regular quadratic algebra for the associated graded ring; these are the socalled gaugc algebras obtaining their name from special examples appearing in $\mathrm{E}$. Witten's gauge theories. The paper surveys basic definitions and properties but concentrates on the development of several concrete examples.
\end{abstract}

\section{0 . Introduction}

Specific problems in defining a "scheme" structure on $\operatorname{Proj}(\widetilde{W})$, where $\widetilde{W}$ is the 4-dimensional quantum space of the Rees ring of the Witten gauge algebras, may be tackled by first introducing such a scheme structure on $\operatorname{Proj}(G(W))$ where $G(W)$ is the associated graded ring of $W$ and then trying to lift this structure to a scheme structure on $\operatorname{Proj}(\widetilde{W})$. In [LVW] this lifting problem is solved by using quantum sections introduced by the second author in [VOS], [RVO] and this explains why the development of the theory of gauge algebras here goes hand in hand with that of quantum sections. In fact Noetherian gauge algebras are particular Zariski rings in the sense of $[$ LVO $, 1,2, \ldots]$. Now quantum sections arise in the sheaf of filtration degree zero of a microstructure sheaf of a Zariski ring over the projective scheme associated to the associated graded ring that is supposed to be commutative in [VOS]. The commutativity of the associated graded ring is nowhere essential in the structure theory of the rings of sections of those sheaves, except of course

\footnotetext{
*This author is supported by an NFWO-grant
} 
in the definition of the topological space and the scheme structure of Proj. However, M. Artin has recently introduced in [Art] the quantum projective space of a quantum $n$-space given by its graded quadratic algebra $Q$ as $\operatorname{Proj}(Q)=Q$-gr $/ \mathcal{F}$, where $\mathcal{F}$ is the full subcategory of graded finite length modules, together with a suitable shift-operator. From this point of view it is natural to try to combine the techniques of Zariski filtered rings inherent in the study of quantum sections with the theory of projective quantum spaces. This is done by introducing the class of positively filtered rings having a non-commutative regular in the sense of Artin and Schelter [AS] quadratic algebra in the sense of Manin, [Man], for the associated graded ring. The restriction to positive filtrations is not essential because the definition of the underlying projective scheme may casily be modified to deal with this; nevertheless we do restrict to the positive case here. The algebras roughly defined above are called gauge algebras. Since the ingredients of the theory lay spread out over several sources not all equally available, we have conceived this paper as a survey paper introducing necessary basic dcfinitions and properties as well as expanding a few concrete examples. A more extensive study of gauge algebras is undertaken by the first author in [LB]; for recent results on formal quantum sections over formal schemes we refer to [RVO].

\section{Filtered rings and associated graded rings}

All rings are associative with unit. A filtration $F R$ on a ring $R$ is given by an ascending chain of additive subgoups $F_{n} R, n \in \mathbb{Z}$, satisfying $I \in F_{0} R, F_{n} R F_{m} R \subset F_{n+m} R$ for $m, n \in \mathbb{Z}$. We always assume that the filtrations considered are exhaustive, that is $\cup_{n \in \mathbb{Z}} F_{n} R=R$, and separated, that is $\cap_{n \in \mathbb{Z}} F_{n} R=0$. The obvious operations induced on the abelian additive group $G(R)=\oplus_{n \in \mathbb{Z}} F_{n} R / F_{n-1} R$ make $G(R)$ into a graded ring with $G(R)_{n}=F_{n} R / F_{n-1} R, n \in \mathbb{Z}$. The principal symbol $\operatorname{map} \sigma: R \rightarrow G(R)$ is defined by putting $\sigma(x)=x \bmod F_{n-1} R$ where $n$ is such that $x \in F_{n} R-F_{n-1} R$. The Rees ring $\widetilde{R}=\oplus_{n \in \mathbb{Z}} F_{n} R$ may be identified with the subring $\sum_{n \in \mathbb{Z}} F_{n} R X^{n}$ of the polynomial ring $R\left[X, X^{-1}\right]$. The notion of the Rees ring for a $\mathbb{Z}$-filtration extends in a natural way the notion of the blow-up ring for an $I$-adic filtration used in singularity theory and commutative Zariski rings. A filtration $F R$ is complete when Cauchy-sequences converge in $R$, or equivalently $R=\underbrace{\lim }_{n} R / F_{n} R$; when $F_{u} R=0$ for $n<0$ then we say that $F R$ is positive or $R$ is positively filtered and it is clear that positive filtrations are complete. Complete filtered rings having a Noetherian associated graded ring are an important class of Zariski rings in the sense of [LVO1], in particular such a ring $R$ has a Noctherian Rees ring $\widetilde{R}$. 
Example 1. The nth Weyl algebra $A_{n}(\mathbb{C})$ is the algebra generated by $2 n$ indeterminates $x_{1}, \ldots, x_{n}$ and $y_{1}, \ldots, y_{n}$ satisfying the commutator relations: $\left[x_{i}, x_{j}\right]=\left[y_{i}, y_{j}\right]=0,\left[x_{i}, y_{j}\right]=\delta_{i j}$. Historically, $A_{n}(\mathbb{C})$ has been introduced as the operator algebra generated by the components $\widehat{x}_{i}$ of the position vector and the components $\widehat{p}_{j}=i \hbar y_{j}$ of the momentum vector of a quantum particle in $n$-dimensional space. The non-vanishing of the commutator $\left[\widehat{x}_{j}, \widehat{p}_{j}\right]=i \hbar$ expresses that one cannot have simultaneous knowledge of position and momentum of a particle in the quantum case. The associated graded ring $G\left(A_{n}(\mathbb{C})\right)$ is the polynomial ring $\mathbb{C}\left[x_{1}, \ldots, x_{n}, y_{1}, \ldots, y_{n}\right]$ that is the operator algebra of the classical (i.e. non-quantum) situation. So in a sense the filtered data may be viewed as quantizations of the associated graded data. The Recs ring $A_{n}(\mathbb{C})^{\sim}$ is the positively graded algebra generated by the degree one element $X$ and $x_{i} X, y_{i} X, 1 \leq i \leq n$ satisfying the commutative relations: $\left[x_{i}, x_{j}\right] X^{2}=\left[y_{i}, y_{j}\right] X^{2}=0$ and $\left[x_{i}, y_{j}\right] X^{2}=\partial_{i j} X^{2}$.

Since $X$ is central in $A_{n}(\mathbb{C})^{\sim}$ we may substitute now indeterminates $X=X, X_{2}=x_{i} X, Y_{i}=y_{i} X$ satisfying homogeneous relations $\left[X_{i}, X_{j}\right]=$ $\left[Y_{i}, Y_{j}\right]=0$ and $\left[X_{i}, Y_{j}\right]=\delta_{i j} X^{2}$. Therefore we may view $A_{n}(\mathbb{C})^{\sim}$ as a quadratic extension of the enveloping algebra of the Heisenberg algebra (see below). Note that we may specialize $X$ to $I$ and we obtain $A_{n}(\mathbb{C})$ a s a specialization of its Rees ring; moreover specializing $X$ to 0 yields $G\left(A_{n}(\mathbb{C})\right)$ as a specialization.

1.1. Lemma. If we let $X$ stand for the canonical homogeneous central regular element of degree one in $\widetilde{R}$ then:

a. $\widetilde{R} /(1-X) \widetilde{R}=R$

b. $\widetilde{R} / X \widetilde{R}=G(R)$.

The observations in the lemma express that $R$ is a "deformation" of $G(R)$ via the Rees ring $\tilde{R}$. Note that for any graded ring with a central regular homogeneous element the construction in a in the lemma yiclds the dehomogenized filtration corresponding to a gradation, cf. [LVO2]. A special case of this dehomogenization principle is well-known in projective algebraic geometry (affine models) and it is also evident in the relation between determinental rings and Schubert cycles.

Example 2. Let $g$ be a finite dimensional Lie algebra, say $g=\mathbb{C} x_{1}+$ $\ldots+\mathbb{C} x_{n}$ with defining relations $\left[x_{i}, x_{j}\right]=\sum \alpha_{i j}^{k} x_{k}$ satisfying the Jacobi identity. By definition all commutators drop in filtration degree for the usual fittration defined on the universal enveloping algebra $U(g)$. One easily checks that $G(U(g)) \cong \mathbb{C}\left[x_{1}, \ldots, x_{n}\right]$ (Poincaré, Birkhof, Witt). The Rees ring $U(g)^{\sim}$ is generated by $X, X_{1}=x_{1} X, \ldots, X_{n}=x_{n} X$, 
satisfying $=\left[X_{i}, X_{j}\right]=\left[x_{i}, x_{j}\right] X^{2}=\sum_{k} \alpha_{i j}^{k} X_{k} X$. Let us provide a more concrete example and consider the 2-dimcnsional non-Abelian Lie algebra $g=\mathbb{C} x+\mathbb{C} y$ satisfying $[x, y]=x$. Then $U(g)^{\sim}$ is the regular algebra in the sense of Artin-Schelter [AS] of [ATV1-2] generated by $X=x Z, Y=y Z$ and $Z$ (now $Z$ plays the role of the central element $X$ before) satisfying the quadratic relations : $X Z-Z X=0, Y Z-Z Y=0$, $X Y-Y X-X Z=0$. We point out that in the classification of [ATV1] this algebra is of type $S_{1}$, in particular it is not of generic, i.e. elliptic, type. The latter property is one that $U(g)^{\sim}$ will share with all other gauge algebras defined later in this paper. Similarly, when $g=\mathrm{sl}_{2}$, $\mathrm{sl}_{2}=\mathbb{C} x+\mathbb{C} y+\mathbb{C} z$ with $[x, y]=2 y,[x, z]=-2 z,[y, z]=x$ then the Rees algebras is the 4-dimensional quadratic algebra (or quantum space) generated by $X=x T, Y=y T, Z=2 T$ and $T$ (now playing the role of the central element of degree onc), satisfying: $X T-T X=0$, $Y T-T Y=0, Z T-T Z=0, X Y-Y X-2 Y T=0, X Z-Z X+2 Z T=0$, $Y Z-Z Y-X T=0$. But again $u\left(\mathrm{sl}_{2}\right)^{\sim}$ is not a Sklyanin algebra in the sense of $[\mathbf{S S}]$.

Most of the algebras we shall consider in this paper will be positively filtered however some localizations of these will be of interest too and so non-positive filtrations will appear naturally. Extreme amongst the nonpositive filtrations are the so-called strongly filtered rings. The filtration $F R$ is said to be strong if $F_{n} R F_{m} R=F_{n+m} R$ holds for every $n, m \in \mathbb{Z}$. It is easy to check that $F R$ is strong if and only if $G(R)$ is a strongly graded ring, i.c. $G(R)_{n} G(R)_{m}=G(R)_{n+m}$ for $n, m \in \mathbb{Z}$; or if and only if $\widetilde{R}$ is a strongly graded ring. We say that $G(R)$ is $d$-strongly graded if $G(R)_{n d} G(R)_{m d}=G(R)_{(n+m) d}$ for $n, m \in \mathbb{Z}$, and $d$ and is minimal as such. For a commutative positively graded ring $A, \operatorname{Proj}(A)$ is locally strongly graded in the sense that for any Zariski open $U \subset \operatorname{Proj}(A)$ the graded ring of sections is $d$-strongly graded for some $d$ (depending on $U)$ : when $A$ is generated over $A_{0}$ by $A_{1}$ as a ring then it is even locally strongly graded because every graded ring of sections will contain a unit of degree one. Kashiwara's ring of germs of micro-differential opcrators on holonomic functions provides an interesting example of a strongly filtered ring.

Example 3. Let $z=\left(z_{1}, \ldots, z_{n}\right)$ be coordinates in $\mathbb{C}^{n}$ and $\xi=$ $\left(\xi_{1}, \ldots, \xi_{n}\right)$ the coordinates for cotangent vectors. Put $T_{0}^{*}\left(\mathbb{C}^{n}\right)=\{(z, \xi)$, $\xi \neq 0\}$, this is an open subset of $\mathbb{C}^{2 n}$ and $z_{1}, \ldots, z_{n}, \xi_{1}, \ldots, \xi_{n}$ are holomorphic functions on $T_{0}^{*}\left(\mathbb{C}^{n}\right)$. Take $p=\left(z^{*}, \xi^{*}\right) \in T_{0}^{*}\left(\mathbb{C}^{n}\right)$. Let $O_{p}$ be the local ring of germs of holomorphic functions (isomorphic to the local ring of convergent power series in $2 n$ variables. Following J.E. Björk (p. 136, [B]) we let $O_{p}(m)$ be the $\xi$-homogeneous elements of order $m$. 
If $U$ is an open set in $T_{0}^{*}\left(\mathbb{C}^{n}\right)$ then $O(m)(U)$ is the set of holomorphic functions in $U$ which are $\xi$-homogeneous of order $m$.

The ring $\mathcal{E}_{p}$ is consisting of the $\Sigma f_{\nu}(z, \xi)$ such that $f_{\nu} \in O(\nu)(U)$ for some open neighbourhood $U$ of $p$ and satisfying the conditions.

i. $f_{\nu}=0$ for all $\nu>w$, for a certain integer $w$.

ii. There exist constants $A$ and $\kappa$ such that

$$
\left|f_{\nu}\right|_{U}=\sup \left\{\mid f_{\nu}(z, \xi),(z, \xi) \in U\right\} \leq A(|\nu| \xi) K^{|\nu|}
$$

for all $\nu$. If $F=\Sigma f_{\nu}, G=\Sigma g_{\mu} \in \mathcal{E}_{p}$ then $F G=\Sigma(\alpha f) \frac{\partial^{\alpha} f_{\nu}}{\partial \xi^{\alpha}} \frac{\partial^{\alpha} g_{\mu}}{\partial z^{\alpha}}$ in multi-index notation.

Now, if $F=\Sigma f_{v} \in \mathcal{E}_{p}$, then the unique largest $w \in \mathbb{Z}$ such that $f_{w} \neq 0$ is called the order of $F$; this defines the filtration of $\mathcal{E}_{p}$ and $\sigma(F)=f_{w}$ is then the principal symbol. With respect to this filtration $G\left(\mathcal{E}_{p}\right)=O_{2 n-1}\left[T, T^{-1}\right]$ is a strongly graded ring which is moreover a regular Noetherian ring of pure dimension $2 n$. The fact that $\mathcal{E}_{p}$ is a Zariski ring (see Section 4) entails all the desired properties for $\mathcal{E}_{p}$.

Although we are mainly concerned with rings here it is useful to establish the corresponding module theory as well. An $R$-module $M$ is filtered if there is an ascending chain of additive subgroups $F_{n} M, n \in \mathbb{Z}$, satisfying $F_{n} R F_{m} M \subset F_{n+m} M$ for $n, m \in \mathbb{Z}$. The category $R$-filt is obtained by taking the filtered $R$-modules and the $R$-linear maps preserving filtration degree for the objects and morphisms. We write $F M$ for the filtration of $M$ and $G(M)=\oplus_{n \in \mathbb{Z}} F_{n} M / F_{n-1} M$ for the associated graded $G(R)$-module. The Grothendieck category of graded $G(R)$ modules will be denoted by $G(R)$-gr. Similarly, we may define the Rees module $\vec{M}$ of $F M$ by $\widetilde{M}=\oplus_{n \in \mathbb{Z}} F_{n} M$ and identify it with a submodule of $M\left[X, X^{-1}\right]$. Again we always assume that $F M$ is exhaustive, $M=U_{n} M$ and separated i.e. $\cap_{n} F_{n} M=0$. We may extend Lemma 1.1. to : $\widetilde{M} / X \widetilde{M} \cong G(M), \widetilde{M} /(1-X) \widetilde{M} \cong M, \widetilde{M}(X)=M\left[X, X^{-1}\right]$ where $(-)_{(X)}$ stands for the object localized at the central multiplicative set of homogeneous elements $\left\{1, X, X^{2}, \ldots\right\}$. In $\widetilde{R}$-gr we have a full subcategory $\mathcal{F}_{X}$ consisting of the $X$-torsionfree graded $\widetilde{R}$-modules.

1.2. Lemma. The functor $\sim: R$-filt $\rightarrow \widetilde{R}$-gr defines on equivalence of categories between $R$-filt and $\mathcal{F}_{X}$. The filtered morphism in $R$-filt corresponding to the morphisms in $\mathcal{F}_{X}$ are the strict morphisms (recall that a filtered morphism $f: M \rightarrow N$ is strict if $F_{N} N \cap \operatorname{Imf}=f\left(F_{n} M\right)$ ). The functor $G: R$-filt $\rightarrow G(R)$-gr is not really exact but it is exact on strict morphisms and sequences of strict morphisms; the functor $D: \widetilde{R}$ $g r \rightarrow R$-filt $\vec{M} \mapsto \vec{M} /(X-1) \widetilde{M}$, is exact. We may define a principal 
symbol map $\sigma_{M}: M \rightarrow G(M)$ by putting $\sigma_{M}(m)=m \bmod F_{n-1} M$ when $m \in F_{n} M-F_{n-1} M$. For $r \in R$ such that $\sigma_{R}(\sigma) \sigma_{M}(m) \neq 0$ we have $\sigma_{R}(r) \sigma_{M}(m)=\sigma_{M}(r m)$. In particular, when $G(R)$ is a domain then $\sigma=\sigma_{R}$ is multiplicative.

A filtration $F M$ is said to be a good filtration if there exist $m_{1}, \ldots, m_{t} \in M$ and $d_{1}, \ldots, d_{t} \in \mathbb{Z}$ such that for all $n \in \mathbb{Z}, F_{n} M=$ $\sum_{i=1}^{t} F_{n-d_{i}} R m_{i}$. The utility of the Rees objects is that properties concerning the filtration $F M$ are translated to properties in $\mathcal{F}_{X}$ concerning the $X$-adic filtration on $\widetilde{M}$.

\subsection{Proposition. With notation as before:}

a. $F M$ is separated if and only if $\vec{M}$ is $X$-adically separated.

b. $F M$ is good if and only if $\vec{M}$ is finitely generated.

c. $F_{-1} R \subset J\left(F_{0} R\right)$ if and only if $X \in J^{g}(\widetilde{R})$, where $J^{g}(-)$ stands for the graded Jacobson radical, cf. [NVO].

d. $F M$ is complete if and only if $\vec{M}$ is $X$-adically complete

e. $F M$ is projective if and only if $\vec{M}$ is projective (similar for fatness).

f. A map $f: M \rightarrow N$ is strict if and only if Coker $\tilde{f} \in \mathcal{F}_{X}$.

\section{Quantum sections as deformations of localizations}

In this section we restrict attention to positively filtered rings $R$ having a commutative Noetherian domain for the associated graded ring. The essential part of this section can and will be considerably generalized in a further section (for Zariskian filtrations).

Consider a multiplicatively closed set $S$ in $R, 1 \in S, 0 \notin S$, such that $\sigma(S)$ is multiplicatively closed in $G(R)$ (this holds automatically when $G(R)$ is a domain because $\sigma$ is multiplicative in that case). Since $\sigma(S)$ consists of homogeneous elements, $\sigma(S)^{-1} G(R)$ is a graded ring. In general $S$ is not an Ore set and so one camnot necessarily form $S^{-1} R$. This draw-back may be overcome by introducing the algebraic microlocalization of $R$ at $\sigma(S)$, we follow the ideas of [AVV].

To the set $S$ we associate the multiplicatively closed set $\widetilde{S}$ in $\widetilde{R}, \widetilde{S}=$ $\left\{\tilde{s}, \tilde{s}=s X^{n} \in \widetilde{R}_{n}\right.$ for $s \in S$ such that $\left.s \in F_{n} R-F_{n-1} R\right\}$. Clearly $1 \in \tilde{S}$, $0 \notin \widetilde{S}$ and $\widetilde{S}$ consists of homogeneous elements of $\widetilde{R}$. For $n \in \mathbb{N}$ there is a canonical epimorphism of graded rings : $\widetilde{\pi}_{n}, \widetilde{R} / X^{n} \widetilde{R} \rightarrow \widetilde{R} / X \widetilde{R} \cong G(R)$. Let $\widetilde{S}(n)$ be the image of $\widetilde{S}$ in $\widetilde{R} / X^{n} \widetilde{R}$. Now ker $\widetilde{\pi}_{n}$ is nilpotent of index $n$ and $\tilde{\pi}_{n}(\widetilde{S}(n))=\sigma(S)$ is an Ore set in $G(R)$, hence $\widetilde{S}(n)$ is an Ore set in 
$\widetilde{R} / X^{n} \tilde{R}$. Therefore we can define : $\widetilde{B}=\widetilde{Q}_{S}^{\mu}(R)=\underbrace{\lim ^{g}}_{n} \tilde{S}(n)^{-1}\left(\tilde{R} / X^{n} \tilde{R}\right)$, where $\underbrace{\lim }_{g}$ stands for the graded inverse limit (the direct sum of the inverse limits of the systems obtained in each degree for the gradation).

2.1. Lemma. The canonical map $\tilde{j}_{S}=\widetilde{R} \hookrightarrow \widetilde{B}$ is a graded ring morphism. We have $\widetilde{B} \in \mathcal{F}_{X}$ and also $\widetilde{B} / \widetilde{j}_{S}(\widetilde{R}) \in \mathcal{F}_{X}$, moreover $\widetilde{B} / X \widetilde{B} \cong \sigma(S)^{-1} G(R)$, cf. [AVV].

After this lemma we may take the dehomogenization of $\widetilde{B}, Q_{S}^{\mu}(R)=$ $\widetilde{B} /(1-X) \widetilde{B}$ and we obtain a filtered ring $Q_{S}^{\mu}(R)$ such that $j_{S}: R \rightarrow$ $Q_{S}^{\mu}(R)$ is a strict filtered morphism, that is the filtration of $R$ is induced by the filtration of $B=Q_{S}^{\mu}(R)$, and $G(B) \cong \sigma(S)^{-1} G(R)$. That $Q_{S}^{\mu}(R)$ is complete (but not positively filtered) is easily veryfied. In fact $Q_{S}^{\mu}(R)$ is nothing but the micro-localization at $\sigma(S)$ as defined by T. Springer in $[\mathbf{S} \mathrm{pr}]$. This follows from the fact that $B$ has the universal property mentioned in the following.

2.2. Lemma. For $s \in S, j_{S}(s)$ is invertible in $B$ and if $B^{\prime}$ is another filtered ring such that $F B^{\prime}$ is complete, $j_{S}^{\prime}: R \rightarrow B^{\prime}$ is a strict inclusion and for every $s \in S$ with $\sigma(s) \in G(R)_{n}$ we have $s^{-1} \in B^{\prime}$ with $\sigma\left(s^{-1}\right) \in$ $G\left(B^{\prime}\right)_{-n}$, then there exists a strict factorization $h: B \rightarrow B^{\prime}$ such that $h j_{S}=j_{S^{\prime}}(c f .[\mathbf{A V V}])$.

Since $Q_{S}^{\mu}(R)$ is complete and $G\left(Q_{S}^{\mu}(R)\right) \cong \sigma(S)^{-1} G(R)$ is a commutative Noetherian domain, this ring will be in the class of rings we consider (though not positively filtered). We may view $Q_{S}^{\mu}(R)$ as a "deformation" of $\sigma(S)^{-1} G(R)$ via the corresponding Rees ring.

When considering the structure of $\operatorname{Proj}(G(R))$ we have to restrict attention to the part of degree zero of $\sigma(S)^{-1} G(R)$, say $G(R)_{(\sigma(S))}=$ $\left(\sigma(S)^{-1} G(R)\right)_{0}$. In particular we have : $F_{0} Q_{S}^{\mu}(R) / F_{-1} Q_{S}^{\mu}(R)=$ $G(R)_{\sigma(S))}$. We define the quantum sections of $R$ at $S$ to the ring $F_{0} Q_{S}^{\mu}(R)=\tilde{Q}_{S}^{\mu}(\tilde{R})_{0}$ equiped with the induced filtration. We denote this ring by $R_{(S)}$. The $F$-saturation of $S$ is $\bar{S}=\{r \in R, \sigma(r) \in \sigma(S)\}$. It is clear that $\bar{S}$ is multiplicatively closed, $1 \in \bar{S}, 0 \notin \bar{S}$, and $\sigma(\bar{S})=\sigma(S)$.

2.3. Proposition. With notation and conventions as before we have: $Q_{S}^{\mu}(R)=Q_{\bar{S}}^{\mu}(R)$. Moreover; $\bar{S}$ is an Ore set in $R$ and for the localized filtration on $(\bar{S})^{-1} R$ (being the one induced on it from $Q_{S}^{\mu}(R)$ ) we have that $Q_{S}^{\mu}(R)=\left((\bar{S})^{-1} R\right)^{\wedge}$, i.e. the microlocalization can always be obtained as a classical localization followed by a completion. 
Combining Proposition 2.3. with foregoing properties and Proposition 1.3.d. we know for a saturated $S$, i.e. $S=\vec{S} Q_{S}^{\mu}(R)^{\sim}: \widetilde{Q}_{S}^{\mu}(\widetilde{R})$ is the graded $X$-adic completion of $(\widetilde{S})^{-1} \widetilde{R}$. The definition of the graded completion yields that

$$
\begin{aligned}
\widetilde{Q}_{S}^{\mu}(\widetilde{R})_{0} & =\underbrace{\lim }_{n}\left((\tilde{S})^{-1} \widetilde{R} / X^{n}(\tilde{S})^{-1} \widetilde{R}\right)_{0}) \\
& =\underbrace{\lim }_{n}\left((\tilde{S})^{-1} \widetilde{R}\right)_{0} /\left(X^{n}(\tilde{S})^{-1} \widetilde{R}\right)_{0}) \\
& =\underbrace{\lim }_{n}\left((\widetilde{S})^{-1} \widetilde{R}\right)_{0} / X^{n}\left((\widetilde{S})^{-1} \widetilde{R}\right)_{-n}) \\
& \cong \underbrace{\lim }_{n} F_{0} S^{-1} R / F_{-n} S^{-1} R=\left(F_{0} S^{-1} R\right)^{\wedge}
\end{aligned}
$$

where $A$ stands for the completion with respect to the filtration induced $F S^{-1} R$ (or by $F Q_{S}^{\mu}(R)$ ) in $F_{0} S^{-1} R$. This provides a way to calculate quantum sections effectively by first calculating $\left(\widetilde{S}^{-1} \widetilde{R}\right)_{0}$ and then allowing the suitable completion.

Example 4. [RVO] Consider the first Weyl algebra $R=A_{1}(\mathbb{C})$. From the foregoing section we know that $G\left(A_{1}(\mathbb{C})\right)=\mathbb{C}[X, y]$ and $A_{1}(\mathbb{C})^{\sim} \cong$ $\mathbb{C}[X, Y, Z] /\left(X Y-Y X-X^{2}, Y Z-Z Y, X Z-Z X\right)$, where we have put $X=x Z, Y=y Z, Z$ being the regular central homogenous element of degree one. Consider $S=\left\{1, x, x^{2} \ldots\right\}$ in $\mathbb{C}[x, y]$. The saturation $\bar{S}$ of $S$ consists of all elements $f$ such that $\sigma(f)=X^{n}$ for some $n$ and this is an Ore set. Note that all elements of the form $\lambda+x, \lambda \in \mathbb{C}$, are contained in $\bar{S}$. The homogeneous Ore set is $\widetilde{S}=\left\{f Z^{n}, f=x^{n}+\sum_{k+l<n} \alpha_{k l} x^{k} y^{l}\right\}$ and we may write $f Z^{n}$ as a homogeneous form of degree $n$ in the new variable: $X=x Z, Y=y Z$ and $Z$, e.g. $x^{2}+y+1$ is lifted to $X^{2}+Y Z+Z^{2}$. In $\left(\tilde{S}^{-1} \tilde{R}\right)_{0}$ we find the elements $Y X^{-1}, X^{-1} Y, Z X^{-1}, X^{-1} Z$ and these satisfy the relations: $Y X^{-1}-X^{-1} Y=\left(Z X^{-1}\right)^{2}, Z X^{-1}-X^{-1} Z=$ 0 . So we have to complete the commuting relations of the generators $p=Y X^{-1}$ and $q=Z X^{-1}$. Now $q p=Z X^{-1} Y X^{-1}=Z\left(Y X^{-1}-\right.$ $\left.Z^{2} X^{-2}\right) X^{-1}=Y Z X^{-2}-Z^{3} X^{-3}=X Y^{-1} Z X^{-1}-Z^{3} X^{-3}=p q-q^{3}$. Hence we arrive at $[p, q]=q^{3}$. Note that $Z \in J^{g}(\widetilde{R})$ hence $Z \in J^{9}\left(\widetilde{S}^{-1} \widetilde{R}\right)$ and therefore every element of the form $X^{n}+Z F_{n-1}(X, Y, Z)$, where $F_{n-1}(X, Y, Z)$ is homogeneous of degree $n-1$, has to yield an invertible $1+Z X^{-1}\left(X^{1-n} F_{n-1}(X, Y, Z)\right)$ in the completion of $\left(\widetilde{S}^{-1} \widetilde{R}\right)_{0}$ because $Z X^{-1} \in J^{g}\left(\widetilde{S}^{-1} \widetilde{R}\right)_{0}, X^{1-n} F_{n-1}(X, Y, Z) \in\left(\widetilde{S}^{-1} \widetilde{R}\right)_{0}$. Therefore we may consider the algcbra $\mathbb{C}\langle p, q\rangle /\left(p q-q p=q^{3}\right)$ as determining the quantum sections up to completion. 
Example 5. Consider the two-dimensional Lie algebra $g=\mathbb{C} x+\mathbb{C} y$ with $[x, y]=x$. Then $U(g)^{\sim}$ is the quadratic algebra generated by $X, Y, Z$ satisfying : $X Z-Z X=0, Y Z-Z Y=0, X Y-Y X=X Z$. Let $\sigma(S)$ be $\left\{1, y, y^{2}, \ldots\right\}$ in $\mathbb{C}[x, y]=G(U(g))$. The saturated Ore set in $U(g)$ is $\bar{S}=\left\{f=y^{n}+\sum_{k+l<n} \alpha_{k i} x^{k} y^{l}\right\}$ and this lifts to a homogeneous Ore set in $U(g)^{\sim}, \widetilde{S}=\left\{f Z^{n}, f \in \bar{S}\right.$ having $\left.\sigma(f)=y^{n}\right\}$. Some relations in degree zero of $\widetilde{S}^{-1} \cup(g)^{\sim}$ derive from the defining relations above : $Z Y^{-1}=Y^{-1} Z, Y^{-1} X-X Y^{-1}=Y^{-1} X Z Y^{-1}$. Consider the canoncial generators $p=X Y^{-1}$ and $q=Z Y^{-1}$, then we calculate :

$$
\begin{aligned}
q p & =Z Y^{-1} X Y^{-1} \\
& =Z\left(X Y^{-1}+Y^{-1} X Z Y^{-1}\right) Y^{-1} \\
& =X Z Y^{-2}+Z Y^{-1} X Y^{-1} Z Y^{-1} \\
& =p q+q p q .
\end{aligned}
$$

Yielding the rather odd relation $[p, q]=-q p q$. However, since $Y-Z \in \widetilde{S}$ we must invert $1-q$ in the quantum sections so we may rewrite the commutation relation as $q p=p \frac{q}{1-q}$ and so we may look at the skew polynomial ring $\mathbb{C}[[q]][p, \gamma]$ where $\gamma$ is the automorphism defined by $p \mapsto \frac{q}{1-q}$ and see that $\mathbb{C}[[q]][p, \gamma]$ determines the quantum sections of $U(g)$ (note that as in Example 4, it sufficed to invert one element, here $Y$, in order to find up to completion the quantum-sections). One should not conclude from the examples 4 . and 5 . that quantum-sections for some $\sigma(S)$ of the type $\left\{1, a, a^{2}, \ldots\right\}$ are always that easy to obtain.

Example 6. Let $g$ be the Lie algebra $s l_{2}$ and change the $s l_{2}$-basis such that $[Y, Z]=X,[Z, X]=Y$ and $[X, Y]=Z$. Consider the multiplicative set $\sigma(S)=\left\{1, X, X^{2}, \ldots\right\}$. The reader may check that the commutation formulas determining the quantum sections of $U\left(s l_{2}\right)$ at $\sigma(S)$ may be given as:

$$
\begin{aligned}
& {[A, B]=\left(A^{2}+B^{2}+1\right) C} \\
& {[A, C]=A C \frac{C^{2}}{1+C^{2}}+B \frac{C^{2}}{1+C^{2}}} \\
& {[B, C]=B C \frac{C^{2}}{1+C^{2}}-A \frac{C^{2}}{1+C^{2}}}
\end{aligned}
$$

where $A=Y X^{-1}, B=Z X^{-1}$ and $C=T X^{-1}$.

Again it is useful to introduce the quantum section of filtered modules. First, in a way formally similar to the way $Q_{S}^{\mu}(R)$ had been constructed we may define $Q_{S}^{\mu}(M)$ for any separated filtered $R$-module $M$ 
with a $\sigma(S)$-torsionfree $G(M)$. Then $F Q_{S}^{\mu}(M)$ is complete, $G\left(Q_{S}^{\mu}(M)\right)=$ $\sigma(S)^{-1} G(M)$. From [AVV] we recall the following exactness properties of microlocalization with respect to good filtrations (note that the positive case considered in the section is a particular case of the Zariskian case considered in [AVV] and in the consequent section 4.).

2.4. Proposition. For every $M$ with good filtration $F M$ we have that $Q_{S}^{\mu}(M) \cong Q_{S}^{\mu}(R) \otimes_{R} M$ as filtered $R$-modules (and $Q_{S}^{\mu}(R)$-modules) and $Q_{S}^{\mu}(R)$ is a flot right $R$-module. The functor $Q_{S}^{\mu}(-)$ is exact on strict sequences.

When introducing quantum sections the reduction to degree zero presents minor problems. By definition $F_{n} R_{(S)}=R_{(S)}$ for $n \geq 0$ and with respect to the filtration of $R_{(S)}$ the inclusion $\widetilde{R}_{(S)} \subset \widetilde{Q}_{S}^{\mu}(\widetilde{R})$ has the property $\left(\widetilde{R}_{(S)}\right)_{m}=\widetilde{Q}_{S}^{\mu}(\widetilde{R})_{m}$ for $m \leq 0$ and $\left(\widetilde{R}_{(S)}\right)_{n}=\left(\widetilde{R}_{(S)}\right)_{0}=R_{(S)}$, for $n \geq 0$. Since the filtration of $Q_{S}^{\mu}(R)$ is complete, the same is true for $F_{0} Q_{S}^{\mu}(R)$ with the induced filtration. So for any $M$ with good filtration $F M$ we define the quantum sections at $S$ by $M_{(S)}=F_{0} Q_{S}^{\mu}(M)$.

\subsection{Proposition.}

1. $M_{(S)}$ is complete for the filtration induced by $F Q_{S}^{\mu}(M)$.

2. The functor $M \mapsto M_{(S)}$ is exact on strict sequences of good filtrations.

3. Suppose that $\sigma(S) \cap G(R)_{1} \neq \phi$ (this is the case in all geometrical situations usually encountered) then $M_{(S)} \cong R_{(S)} \otimes F_{0} M$.

2.6. Note. It is possible that $M_{(S)}=0$ but $M \neq 0$. This may be expressed in terms of the characteristic variety of $M$, see later. As before we may facilitate notation by putting $G(M)_{(\sigma(S))}=\left(\sigma(S)^{-1} G(M)_{0}\right.$, so $M_{(S)} / F_{-1} M_{(S)}=G(M)_{\sigma(S))}$.

\section{Quantum sections and coherent sheaves over projective schemes}

Assumptions on $R, G(R)$ are as in Section 2. We consider $\operatorname{Proj}(G(R))=$ $Y$ with its Zariski topology. The graded structure sheaf $O_{Y}^{g}$ is defined by associating to a basic open set $Y(f)$ in $Y, f$ homogeneous in $G(R)$, the graded ring of sections $Q_{f}(G(R))=G(R)\left[f^{-1}\right]$, the structure sheaf $\underline{O}_{Y}$ is the part of degre zero sheaf, $\underline{O}_{Y}=\left(\underline{Q}_{Y}^{g}\right)_{0}$. The ringed space $\left(Y, \underline{Q}_{Y}\right)$ is a scheme. The ringed space $\left(Y, Q_{Y}^{g}\right)$ is a coherent Noetherian sheaf of rings. To $p \in \operatorname{Proj}(G(R))$ we associate the multiplicative set $G(R)-p=$ 
$S(p)$, to $Y(f)$ we associate $S(f)=\left\{1, f, f^{2}, \ldots\right\}$. We define the microstructuresheaf of $R$ over $Y$ by associating to $Y(f)$ the ring $Q_{S(f)}^{\mu}(R)$. This defines a sheaf $\underline{O}_{Y}^{\mu}$ and by applying the functor $\sim$ (sectionwise) we obtain the Rees sheaf $\widetilde{O}_{Y}^{\mu}$. One should pay some attention when dealing with these sheaves stalk-wise :

3.1. Proposition. [SVO] $\underline{Q}_{Y}^{\mu}$ and $\widetilde{O}_{Y}^{\mu}$ are sheaves and for each $p \in Y$ the stalk $\underline{O}_{Y, p}^{\mu}$ is a Zariski fittered ring in the sense of [LVO1] (see also Section 4). Moreover the completion of $\underline{O}_{Y, p}^{\mu}$ at its filtration yields exactly the microlocalization $Q_{S(p)}^{\mu}(R)$.

For notational convenience we write $\underline{\mathcal{R}}=\underline{O}_{Y}^{\mu}, \underline{\widetilde{R}}=\underline{O}_{Y}^{\mu}, G(\underline{\mathcal{R}})=$ $\underline{\widetilde{R}} / \underline{X \tilde{\mathcal{R}}}$, where $\underline{X}$ stands for the global section of $\underline{\widetilde{R}}$ determined by $\underline{X} \mid Y(f)=X_{Q_{f}^{\mu}(R)}$. Then $\underline{\tilde{\mathcal{R}}}$ and $G(\underline{\mathcal{R}})$ are graded sheaves and the sheaf $\underline{\mathcal{R}}$ is filtered in the sense that there are subsheaves of groups $\mathcal{F}_{n} \underline{\mathcal{R}}$ given by $\mathcal{F}_{n} \underline{R}(Y(f))=F_{n} Q_{S(f)}^{\mu}(R)$ on the basis $\left\{Y(f), f \in G(R)_{n}\right.$ for some $n>0\}$. Obviously we obtain a subsheaf of rings $\mathcal{F}_{0} R$ given by $\mathcal{F}_{0} \underline{\mathcal{R}}(Y(f))=F_{0} Q_{S(f)}^{\mu}(R)=R_{(S(f))}$. It is clear that $\mathcal{F}_{0} \underline{\mathcal{R}}=(\underline{\tilde{R}})_{0}$ as sheaves over $Y$. For some notions concerning sheaves we may refer to $\mathrm{P}$. Schapira [Schap].

3.2. Proposition. $\mathcal{F}_{0} \underline{\mathcal{R}}$ is a Noetherian coherent sheaf of rings, the stalk at $p \in Y$ is such that $\left(\mathcal{F}_{0} \mathcal{R}\right)_{p}^{\wedge}=R_{[S(P)]}$ where $\wedge$ stands for the completion at the filtration of $\left(\mathcal{F}_{0} \underline{R}\right)_{p}$ defined by $\left(\mathcal{F}_{n} \underline{\mathcal{R}}\right)_{p}, n \leq 0$.

3.3. Theorem. $\mathcal{F}_{0} \mathcal{R}$ is a sheaf of Zariski rings i.e. sections on basic open sets are Zariski rings, having Zariski rings for the stalks too. $\mathcal{F}_{-1} \underline{\mathcal{R}}$ is a Noetherian coherent ideal of $\mathcal{F}_{0} \underline{\mathcal{R}}$ and $\mathcal{F}_{0} \underline{\mathcal{R}} / \mathcal{F}_{-1} \underline{\mathcal{R}}=\underline{O}_{Y}$.

We call $\mathcal{F}_{0} \underline{\mathcal{R}}$ the sheaf of quantum sections over $\underline{O}_{Y}$. Let us mention a fow examples of this.

Example 7. We reconsider the case $R=A_{1}(\mathbb{C})$. First let us point out that it suffices to describe a sheaf on the basis of the topology given by the $Y(f), f$ homogeneous of positive degree in $\mathbb{C}[x, y]$. When checking the proof of the fact that $\underline{O}_{Y}^{\prime \prime}$ is a sheaf one sees that the real localizations at the corresponding Ore sets of $R$, that is the $\overline{S(f)}$, define a subsheaf $\underline{O}_{Y}^{\lambda} \subset \underline{O}_{Y}^{\mu}$ such that $\underline{O}_{Y}^{\mu}$ is the sheaf-wise completion (this is always calculated sectionwise, not stalk-wise !) completion of $\underline{O}_{Y}^{\lambda}$ at the filtration $\mathcal{F} \underline{O}_{Y}^{\lambda}$ sectionwise defined by the localized filtrations in $\overline{S(f)}^{-1} R$. We will describe the quantum sections by describing $\underline{O}_{Y}^{\lambda}$ i.e. up to a 
completion that does not interfere with the commutation relations. Now $\operatorname{Proj}(G(R))=\mathbb{P}^{1}$ in this case and we cover $\mathbb{P}^{1}$ by $Y(x) \cong \operatorname{Spec} \mathbb{C}\left[\frac{y}{x}\right]$ and $Y(y) \cong$ Spec $\mathbb{C}\left[\frac{x}{y}\right]$ corresponding to $S(x)$ and $S(y)$ respectively. Of course $Y(x) \cap Y(y) \cong \mathrm{Spec} \mathbb{C}\left[\frac{x}{y}, \frac{y}{x}\right]$ corresponds to $S(x y)$. Over $S(x), S(y)$ and $S(x y)$ we have to calculate the localizations at the corresponding Ore sets in $R$, this yields the following cases :

$S(x)$ : We have seen before that we obtain a defining relation $[p, q]=q^{3}$ with $p=Y X^{-1}$ and $q=Z X^{-1}$.

$S(y)$ : Using the anti-symmetriy between $x$ and $y$ it is easy to obtain the defining relation $\left[p^{-1}, v\right]=-v^{3}$, where $p^{-1}=X Y^{-1}, v=Z X^{-1}$. Note that $v=q p^{-1}$.

$S(x y)$ : We can glue the above rings together via their embedding in the ring generated by $p, p^{-1}, q$ (or $p, p^{-1}, v$ ).

This may be schematically picturised as follows:

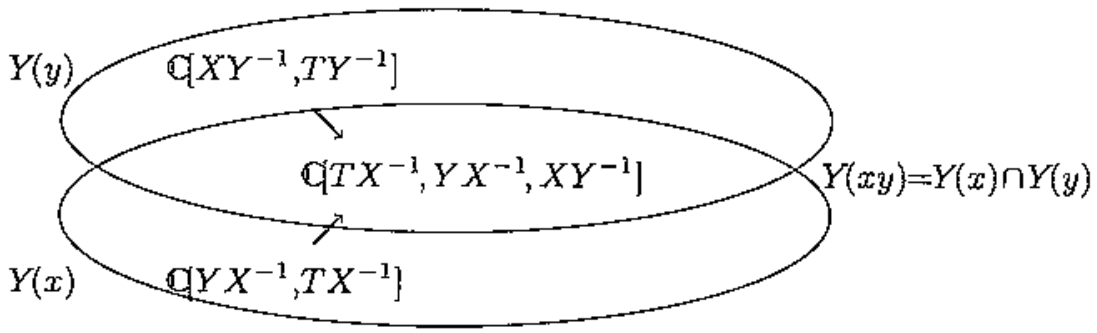

Example 8. We now let $R$ be the enveloping algebra $U(g)$ where $g$ is again the two-dimensional non-A belian Lie algebra considered before. Again Proj $(G(R))=\mathbb{P}^{1}$ and we consider the same covering as in foregoing example.

$S(x)$ : Since $x$ is a normalizing element in $U(g)$ it suffices to localize classically at $\left\{1, x, x^{2}, \ldots\right\}$ in order to describe the quantum sections at $S(x)$ up to completion. The canonical generators are $p=Y X^{-1}$ and $q=Z X^{-1}$ and one calculates: $p q=Y X^{-1} Z X^{-1}=Z Y X^{-2}=Z\left(X^{-1} Y+X^{-1}=q p+q^{2}\right.$, hence $[p, q]=q^{2}$, which defines the exceptional 2-dimensional quantum plane.

$S(y)$ : It is easily seen that we obtain the quantum sections from $\mathbb{C}[[\omega]]\left[p^{-1}, \gamma\right]$ where $\omega=Z X^{-1}$ and $\gamma$ is the automorphism determined by $\omega \mapsto \frac{\omega}{1-\omega}$ (see Example 5 ). 
$S(x y):$ Note that $\omega=q p^{-1}$. The schematic picture obtained is :

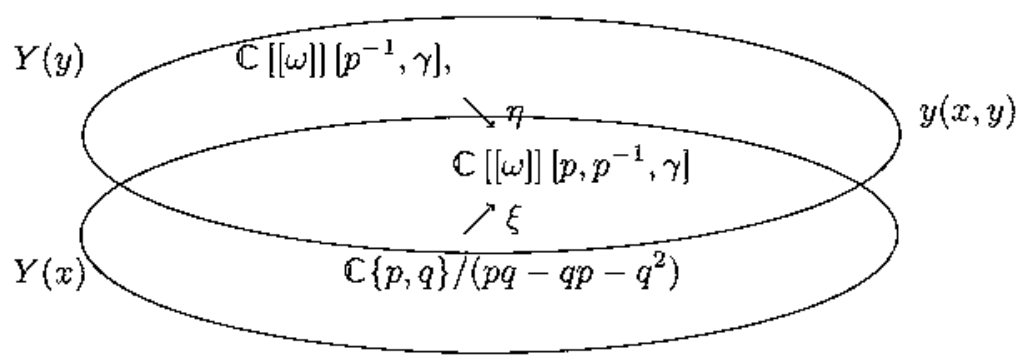

where $\xi$ maps $q$ to $\omega p$ and $\eta$ is clear.

So in constructing the quantum "scheme" of $U(g)$ we glue a 2dimensional quantum space (see section 4) with a very "localized" algebra.

Again we finish this section by providing the corresponding notions for modules with good filtrations. If $F M$ is good then there is a fil-

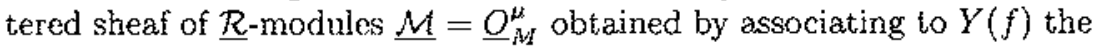
microlocalization $Q_{S(f)}^{\mu}(M)$. We also obtain a shcaf of $G(\underline{\mathcal{R}})$-modules $G(\underline{\mathcal{M}})=\underline{O}_{G(M)}^{g}$. Since the latter is coherent and $\underline{\mathcal{M}}$ is locally finite (because $M$ is finitely generatcd) it follows that $\mathcal{M}$ is coherent. Thercfore we obtain a coherent sheaf $\mathcal{F}_{0} \underline{\mathcal{M}}$ and a coherent $\mathcal{F}_{0} \underline{\mathcal{R}}$-submodule $\mathcal{F}_{-1} \underline{\mathcal{M}}$ such that $\mathcal{F}_{0} \mathcal{M} / \mathcal{F}_{-1} \underline{M}=\underline{O}_{G(M)}$, the usual structure sheaf of $G(M)$ over $\operatorname{Proj}(G(R))$. We refer to $\mathcal{F}_{0} \mathcal{M}$ as the sheaf of quantum sections of $O_{G(M)}$ over Proj $(G(R))$. Quantum sections of pure filtrations are under consideratrion at this moment.

3.3. Proposition. If $M \underset{f}{\rightarrow}$ is a strict filtered morphism then the induced $\mathcal{F}_{0} \underline{M} \underset{\varphi}{\rightarrow} \mathcal{F}_{0} \underline{N}$ is strict in the sense of sheof filtrations. If $F M$ is good then $\underline{\mathcal{F}}$ is good on $\mathcal{F}_{0} \underline{\mathcal{M}}$ in the sheaf sense, [RVO].

For a given $M \in R$-filt with good filtration $F M$ we define: $\mathcal{A}=$ $\operatorname{Ann}_{G(R)}(G(M)), V(\mathcal{A})=V(M) \subset \operatorname{Proj}(G(R))$ the closed subscheme determined by the graded ideal $\mathcal{A}$. It is costumary to call $V(M)$ the characteristic variety of $M$, so we define the quantum sections over the characteristic variety as the restricted sheaf $\mathcal{F}_{0} \mathcal{M} \mid V(M)$ i.e. we associate $M_{(S(f))}$ to $Y(f) \cap V(\mathcal{A})$. When $Y(f) \cap V(\mathcal{A}) \neq \phi$ then $Q_{S(f)}^{\mu}(M) \neq 0$.

So the assumption that the sets $Y(f)$ with $f \in h G(R)$ of non-zero degree form a basis for the Zariski topology leads to $F_{0} Q_{S(f)}^{\mu}(M) \neq 0$ and hence the sheaf $\mathcal{F}_{0} \mathcal{M} \mid V(M)$ has non-vanishing sections everywhere. For more theory concering cohcrent sheaves over micro-structuresheaves 
we refer to [RVO]; let us mention that the notion of a good filtration is replaced in the sheaf theory by the notion "coherently filtered". In [RVO2] quantum sections are viewed as a degenerate case of formal quantum sections, here one is working with formal schemes obtained by formal completions along closed subschemes and the non-commutative "formal" sheaves constructed over them.

\section{Zariski rings to gauge algebras}

The theory of filtered rings, in particular microlocalization, may be extended to the case of non-commutative associated graded rings. Of course, when constructing the microlocalizations one has to start off with an Ore set $\sigma(S)$ in the associated graded ring that can be lifted to a saturated Ore set in the filtered ring as well as to an homogeneous Ore set in the Rees ring. From then on all constructions may be carried out without essential changes. This generality is allowed by the theory of Zariskian filtrations and the consequent use of the Rees objects that has been the guiding philosophy of Li Huishi, F. Van Oystaeyen in [LVO6] and consequent publications. Since the important examples of enveloping algebras and rings of differential operators have commutative associated graded rings it may be necessary to point out that the generelization strived for is not as artificial as it may seem at first sight. Just like the forementioned algebras may be viewed as deformations of polynomial rings via the Rees ring, gauge algebras can be introduced as deformations of quantum spaces. An $n$-dimensional quantum space (or rather its function ring) is an affine positively graded $C$-algebra $Q=\mathbb{C} \oplus Q_{1} \oplus \ldots \oplus Q_{m} \oplus \ldots$ that is a quadratic algebra in the sense of Manin, [Man], i.e. $Q_{1}$ gencrates $Q$ as a ring over $C$ and the defining relations are quadratic, and $Q$ is regular in the sense of Artin, Schelter, [AS], i.e. gldim $Q=G K \operatorname{dim} Q=n$ and $\operatorname{Ext}_{Q}^{i}(k . Q)=.\delta_{i n} \mathbb{C}$. Results of R. Irving, [Irv], for $n=2$ and M. Artin, W. Scheiter, J. Tate, M. Van den Bergh [ATV1-2] for $n=3$ establish a complete classification of quantum spaces for $n \leq 3$; for $n>3$ the classification is non-existing. A gauge algebra $G$ is a positively filtered algebra (of finite global dimension) such that its associated graded ring $Q=G(\mathcal{G})$ is a quantum space. Weyl algebras, eveloping algebras and the rings of differential operators usually considered are gauge algebras, but there are many others.

Example 9. Let $A_{1}(\mathbb{C}, q)$ be the $\mathbb{C}$-algebra generated by $x$ and $y$ satisfying the relation $x y-q y x=1$. It is clear that $G\left(A_{1}(\mathbb{C}, q)\right)=\mathbb{C}[x][y, \tau]=$ $\mathbb{C}[x, y]$ where $\tau(x)=q x$, this skew polynomial ring is the function ring of the quantum plane. The algebra $A_{1}(\mathcal{C}, q)$ is called the quantized Wcyl algebra in [Good]. In $\mathbb{C}_{q}[x, y]$ the set $\left\{1, x, x^{2}, \ldots\right\}$ is an Ore set that 
yields a saturated Ore set $\overline{S(x)}=\left\{f=x^{n}+\sum_{k+l<n} \alpha_{k l} x^{k} y^{l}\right\}$ and a homogeneous Ore set : $\tilde{S}=\left\{\tilde{f}=X^{n}+\sum_{k+l<n} \alpha_{k, l} X^{k} Y^{l} J^{n-k-l}\right\}$ in the Rees ring of $A_{1}(\mathbb{C}, q)$ The Rees ring $A_{1}(\mathbb{C}, q)^{\sim}$ is generated over $\mathbb{C}$ by $X, Y, T$ satisfying $[X, T]=[Y, T]=0$ and $X Y-q Y X=T^{2}$. The quantum sections of $A_{1}(\mathbb{C}, q)$ at $S(x)$ is the completion of $\mathbb{C}$-algebra generated by $A=Y X^{-1}$ and $B=T X^{-1}$ satisfying the commutation relation : $A B-q B A=B^{3}$. After a basechange $A \rightarrow A^{\prime}=(q-1) A+B^{2}$ this strange looking algebra becomes the quantum plane $\mathbb{C}_{q}\left[A^{\prime}, B\right]$.

Example 10. Witten's gauge algebras.

In [Wit], E. Witten provided a partial explanation for the existence of quantum groups. He established that it is possible to summarize the data in Chern-Simons gauge theory with gauge group $G$ in terms of a Hopfalgebra deformation of the universal enveloping algebra of $G$. When $G=S U(2)$ this deformation is equivalent to Woronowich representation of quantum $S U(2)$, cf. [WOR]. The argument then comes down to the statement that quantum Lie Algebras exist because 3-dimensional Chern-Simons gauge theory exist. The general form of Witten's gauge algebras $W$ for $S U(2)$ are the $\mathbb{C}$-algebras generated by $X, Y$ and $Z$ satisfying the relations: $Y X+\alpha X Y+\beta Y=0, Y Z+\gamma Z Y+\delta X^{2}+\varepsilon X=0$, $Z X+\xi X Z+\eta Z=0$. For $G(W)$ we obtain the following defining relations: $Y X+\alpha X Y=0, Y Z+\gamma Z Y+\delta X^{2}=0, Z X+\xi X Z=0$. We see that $G(W)$ is a 3-dimensional quantum space of type $S_{1}^{\prime}$ in the sense of [ATVI] i.e. a line plus conic situation. Note that 3 -dimensional quantum spaces have a canonical normalizing element of degree 3 ; in the above case this element is a product of two normalizing elements, one of degree 1 (being $X$ ) and one of degree 2 having a physical explanation as the deformation of the Casimir operator. This situation turns out to be typical for 3-dimensional gauge algebras, that they aiways have normalizing elements of degree 1 and degree 2 in the associated graded ring and one can microlocalize at these normalizing elements because they generated Ore sets.

In the foregoing sections we focussed on positive filtrations but this is somewhat unsatisfactory firstly because this class is not closed under the operations we consider (localizations etc...) and secondly because several important examples; e.g. I-adic filtrations, germs of microdifferential operators etc..., do not fit in the class. In order to have a class of filtered rings allowing for non-positive filtrations as well as non-commutative associated graded rings but still allowing the use of homological algebra methods in order to deal with regularity of non-commutative rings and global dimension it is necessary to have the following basic properties.

i. If $M$ has good filtration $F M$ and $N$ is a submodule of $M$ equiped with the induced filtration $F N=N \cap F M$ then $F N$ is again a 
good filtration.

ii. Every good filtration is separated.

It turns out that $i$ and ii are equivalent to the following : $\widetilde{R}$ is Noetherian and $X \in J^{g}(\tilde{R})$ (for several equivalent statements we refer to [LVO1], and in this case the filtration $F R$ is said to be Zariskian. Every complete filtered ring $R$ such that $G(R)$ is Noetherian is a Zariskian ring and in particular the positive case with Noetherian associated graded ring is also a particular case. The results of Section 1 and Section 2 remain valid for Zariski rings in general; even for Section 3 one can do a lot but one has to define a suitable topological space first. Note that we will assume the conditions $i$ and ii for both left and right modules, so the Zariski rings mentioned here are left and right Zariski rings, as in [LVO, $1,2]$.

We mention some fundamental results stcmming from $[$ LVO $, 1,2, \ldots]$.

4.1. Theorem. Let $R$ be a Zariski ring. If $G(R)$ has finite global dimension then

i. $\operatorname{gldim} \widetilde{R}=1+\operatorname{gldim} G(R)$

ii. gldim $R \leq \operatorname{grgldim} G(R)=\operatorname{grgldim} \widetilde{R}-1$, where grgldim stands for the gldim in the graded category.

iii. gldim $\widehat{R}=\operatorname{gldim} R$

4.2. Theorem. Let $R$ be a Zariski ring. If $G(R)$ is a regular Noetherian (in the sense of Auslander) then $R$ and $\widetilde{R}$ are regular Noetherian. Recall that a non-commutative ring is regular in the sense of Auslander if it has finite global dimension and every finitely generated left or right module satisfies the Auslander condition; recall that a finitely generated $R$-module $M$ satisfies the Auslonder condition if for every $0 \leq k \leq \mu=\operatorname{gldim} R$ and any nonzero $R$-submodule $N$ of $\operatorname{Ext}_{R}^{k}(M, R)$ we hove $j_{R}(N) \geq k$, where $j_{R}(-)$ stands for the grade number, i.e. the smallest notural number $j$ such that $\operatorname{Ext}_{R}^{j}(-, R) \neq 0$.

4.3. Corollary. If $\mathcal{G}$ is a Noetherian gauge algebra then $\mathcal{G}$ and $\tilde{\mathcal{G}}$ are Noetherian regular algebras. If $G(\mathcal{G})$ is an n-dimensional quantum space then: $\operatorname{gldim} \widetilde{\mathcal{G}}=1+n, \operatorname{gldim} \mathcal{G} \leq n$. Moreover, $\widetilde{G}$ is an $n+1-\operatorname{dim}$ quantumspace.

The "scheme"-theoretic treatment of gauge algebras has its roots in trying to understand the geometry of so-called innocent quantum spaces, i.e. quantum spaces that are Noctherian and posessing a central element $t$ of degree one. The innocent quantum space corresponding to a gaage 
algebra $\mathcal{G}$ is its Rees ring $\widetilde{\mathcal{G}}$. In [Art] the quantum projective space of a quantum $n$-space $Q$ is defined to be $\operatorname{Proj}(Q)=Q$-gr $\mathcal{F}$, where $\mathcal{F}$ is the full subcategory of finite length modules, together with a shift operation [Art, Definition 1.2.]. For a commutative ring, or one that is a finite module over its center, one may recover the underlying scheme structure from Serre's theorem. In general however the "scheme" structure of $\operatorname{Proj}(Q)$ as defined above is far from being understood. A first feeling for the underlying problems can be obtained by considering particular modules, i.e. the point- and line-modules, cf. [ATV2], and the fat point modules introduced in [Art]. If we restrict attention to the geometry of innocent quantum spaces we can use the gauge algebra and its quantum sections to put a "scheme" structure on $\operatorname{Proj}(\widetilde{\mathcal{G}})$ which reduces the study of (fat) point-modules to that of finite dimensional representations of algebras, cf. [LB]. In fact, wo may view $\operatorname{Proj}(\tilde{\mathcal{G}})$ as an affine piece corresponding to the gauge algebra $\mathcal{G}$ and a piece at infinity identified to $\operatorname{Proj}(\tilde{\mathcal{G}} / t \tilde{\mathcal{G}})=\operatorname{Proj}(G(\mathcal{G}))$. That is a lower dimensional projective quantum space. Assume by induction that we have been able to put a scheme structure on $\operatorname{Proj}(G(\mathcal{G}))$ with affine open sets corresponding to some graded Ore sets $S_{1}, \ldots, S_{k}$ and it is not restrictive to assume that each of these Ore sets may be generated by a single element. Then we may cover $\operatorname{Proj}(\widetilde{\mathcal{G}})$ by open sets corresponding to the quantum sections with respect to the $S_{i}, i=1, \ldots, k$ plus the appropriate glueing morphisms. Note that these quantum sections and their "glues" with $\mathcal{G}$ may be viewed as a scheme structure on $\operatorname{Proj}(\tilde{\mathcal{G}})$. Let us provide some easy examples here.

Example 11. Reconsider the first Weyl algebra $A_{1}(\mathbb{C})$. We use the calculations made above to cxtend the schematic picture in Example 7 by glucing to the open sct corresponding to $Y(Z)$ i.e. the affine piece corresponding to $\left(A_{1}(\mathbb{C})\right.$. We obtain the following diagram of glueing data:

$$
\begin{aligned}
& \mathbb{C}\left\{X Z^{-1}, Y Z^{-1}, Z X^{-1}\right\} \hookleftarrow A_{1}(\mathbb{C})=\mathbb{C}\left\{X Z^{-1}, Y Z^{-1}\right\} \hookrightarrow \mathbb{C}\left\{X Z^{-1}, Y Z^{-1}, Z Y^{-1}\right\} \\
& {\left[X Z^{-1}, Y Z^{-1}\right]=1} \\
& \begin{array}{cc}
\mathbb{C}\left\{Y X^{-1}, Z X^{-1}\right\} & \mathbb{C}\left\{X Y^{-1}, Z Y^{-1}\right\} \\
\left\{Y X^{-1}, Z X^{-1}\right]=\left(Z X^{-1}\right)^{3} & {\left[X Y^{-1}, Z Y^{-1}\right\}=\left(Z Y^{-1}\right)^{3}} \\
\mathbb{C}\left\{Z X^{-1}, Y X^{-1}, X Y^{-1}\right\} &
\end{array}
\end{aligned}
$$

To find its point modules we have to study the one-dimensional repre- 
sentations and their glueing data.

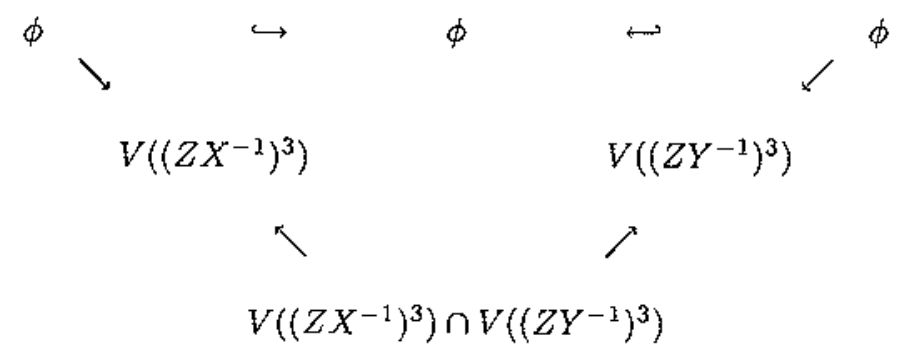

corresponding to the fact that the associated degree 3 divisor of the quantum 3-space $A_{1}(\mathbb{C})^{\sim}$ is $Z^{3}$.

This may be pictured in the usual $\mathrm{P}^{2}$.

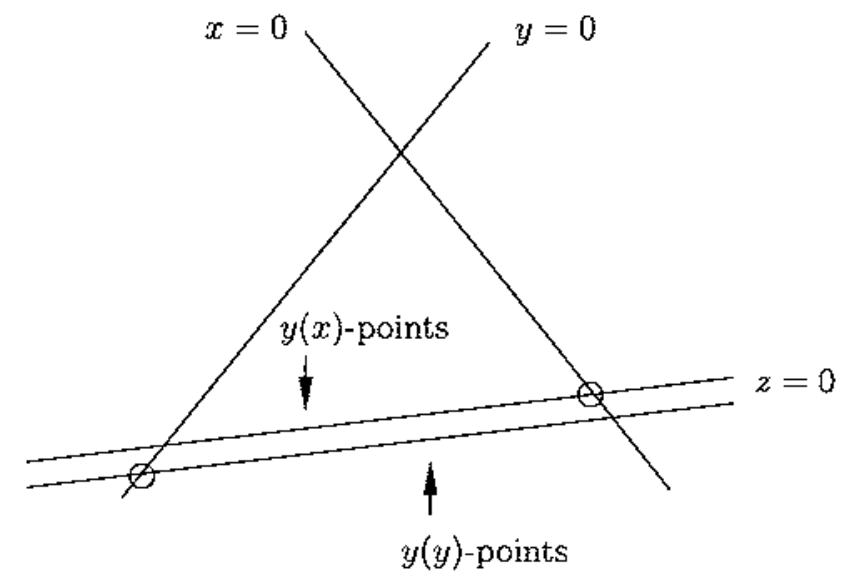

where the circle means that the intersection point is missing and we have drawn a double copy of the samc $z=0$ locus.

Example 12. Reconsider the situation of Example 8. Using the computations and notations of that Example 8, we obtain the following diagram of glueing data: 


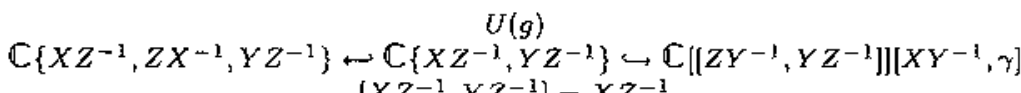

$$
\begin{aligned}
& {\left[X Z^{-1}, Y Z^{-1}\right]=X Z^{-1}}
\end{aligned}
$$

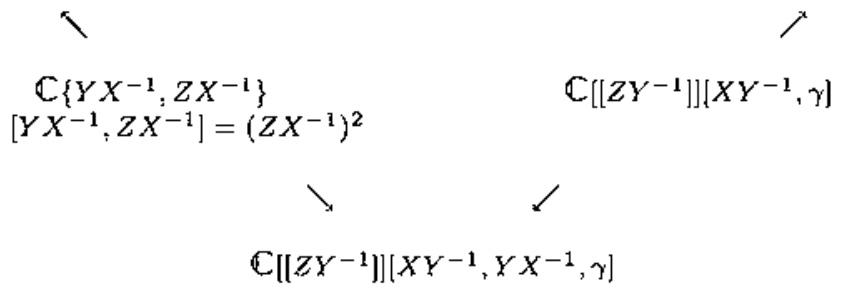

Only the top left corner (glueing the enveloping algebra to the exceptional quantum space) resembles the commutative case. The other two comers have shrunk in dimension. It may be helpful in understanding these phenomena to look at the picture of point-modules in $\operatorname{Proj}(\overrightarrow{U(g)})$ pictured in the usual $\mathbb{P}^{2}$ :

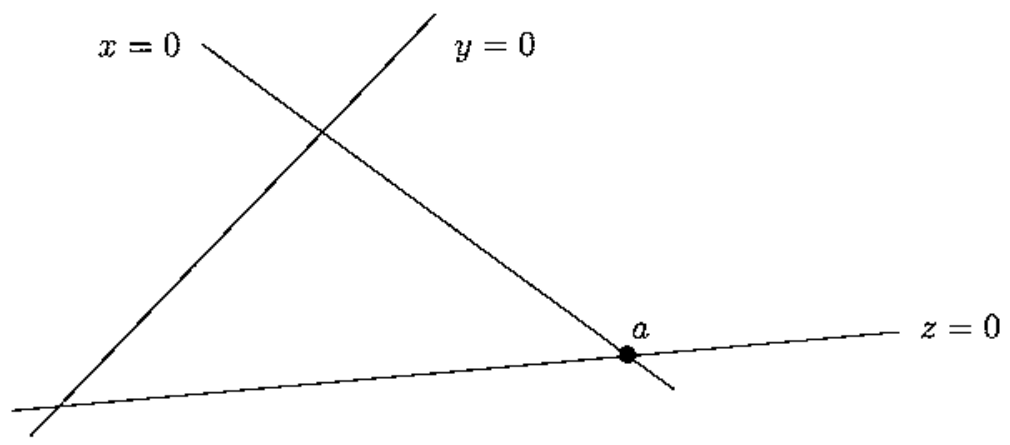

where :

the $y(x)$-points are $z=0$ without the point $a$, the $y(z)$-points are $x=0$ without the point $a$, the $y(y)$-points are $a$.

Example 13. The quantized Weyl algebra $A_{1}(\mathbb{C}, q)$ as a gauge algebra. We use notation and calculations as in Example 9 . Put $R=A_{1}(\mathbb{C}, q)$. Both $X$ and $Y$ are normalizing in the quantum plane $\mathbb{C}_{q}[X, Y]$, so we may define a "scheme" structure on $\operatorname{Proj}(\widetilde{R})$ by giving 
the following glueing data.

$$
\begin{aligned}
& \mathbb{C}\left\{X T^{-1}, T X^{-1}, Y T^{-1}\right\} \hookleftarrow\left\{X T^{-1}, Y T^{-1}\right\} \leftrightarrow \mathbb{C}\left\{X T^{-1}, Y T^{-1}, T Y^{-1}\right\} \\
& X T^{-1}, Y T^{-1}-q Y T^{-1} X T^{-1}=1 \\
& C\left\{Y X^{-1}, T X^{-1}\right\} \\
& C\left\{X Y^{-1}, T Y^{-1}\right\} \\
& Y X^{-1} T X^{-1}-q T X^{-1} Y X^{-1}=\left(T X^{-1}\right)^{3} \quad X Y^{-1} T Y^{-1}-\frac{1}{q} T Y^{-1} X Y^{-1}=-\frac{1}{q}\left(T Y^{-1}\right)^{3} \\
& C\left\{Y X^{-1}, X Y^{-1}, T X^{-1}\right\}
\end{aligned}
$$

and again one can visualize the point modules as points in $p^{2}$. The picture corresponds to the fact that the associatcd degree 3 divisor is $T\left(T^{2}+(q-1) X Y\right)$ :

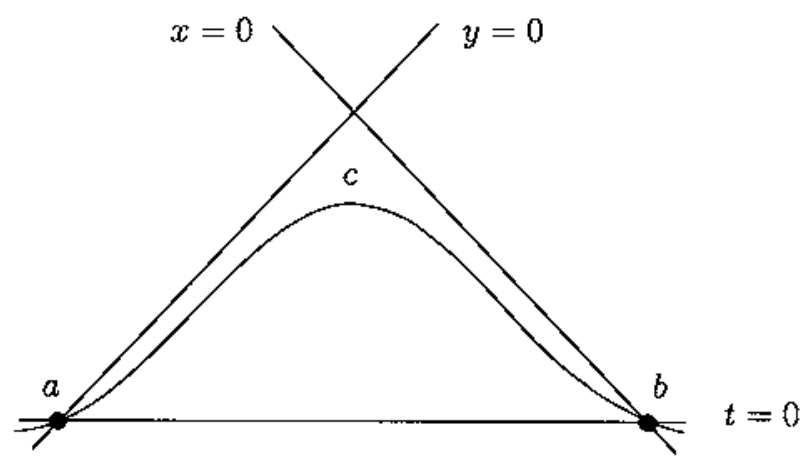

where $C$ is the conic defined by $T^{2}+(q-1) X Y$.

$$
\begin{aligned}
& Y(T) \text { points are } C-\{a, b\} \\
& Y(X) \text { points are } C-\{b\} \cup(T=0)-\{b\} \\
& Y(Y) \text { points are } C-\{a\} \cup(T=0)-\{b\}
\end{aligned}
$$

The scheme structure of $A_{1}(\mathbb{C}, q)^{\sim}$ described above is critical in defining the scheme structure on $\operatorname{Proj}(\widetilde{W})$ where $\widetilde{W}$ is the 4-dimensional quantum space of the Rees ring of the Witten gauge algebras. For, it is possible to change the polarization on $\operatorname{Proj}(G(W))$ as in [Art] to obtain $\operatorname{Proj}\left(A_{1}(\mathbb{C}, q)^{\sim}\right)$ and use the foregoing in order to define a scheme structure on $\operatorname{Proj}(G / W))$ that is then lifted via quantum sections to $\operatorname{Proj}(\widetilde{W})$ as in [LVW]. In a similar way one can study the fat-point medules of multiplicity $n$ (as in [Art]) in an innocent projective quantum space by glueing together the $n$-dimensional representation of the scheme components. 


\section{References}

[Art] M. ARTin, Geometry of Quantum Planes, Preprint MIT (1991). [AS] M. ARTin AND W. Schelter, Graded Algebras of Global Dimension Three, Adv. Math. 66 (1987), 171-216.

[ATV1] M. Artin, J. Tate and M. Van den Bergh, Some Algebras Associated to Automorphisms of Elliptic Curves, in "The Grothendieck Festschrift, Vol. I," Birkhauser (1990), pp. 33-85.

[ATV2] M. ARtin, J. TAte and M. VAN DEN Bergh, Modules over Regular Algebras of Dimension Three, MIT.

[AVV] M.-J. Asensio, M. VAN Den Bergh and F. Van OysTAEYEN, A New Algebraic Approach to Microlocalization of Filtered Rings, Trans. Amer. Math. Soc.

[B] J. E. BJöRK, "Rings of Differential Operators," Math. Library 21, North. Holland, Amsterdam, 1979.

[LB] L. LE BRUYN, Gauge Algebras, In preparation.

[LVO1] Li Huishi AND F. VAN OYSTAEYeN, Zariskian Filtrations, Comm. in Algebra 17(12) (1989), $2945 \cdot 2470$.

[LVO2] Li Huishi ano F. Van Oystaeyen, Global Dimension and Auslander Regularity of Rees Rings, Bull. Soc. Math. Belg.

[LVW] L. Le BRUyn, F. VAN OYstaeyen and L. Willaert, Quantum Sections of Schematic Algebras, UIA preprint, November (1992).

[Man] YU I ManiN, "Quantum GToups and non Commutative Geometry," Publ. Centre Prech. Math. Montréal, 1988.

[RVO1] A. RADWAN AND F. VAN OYSTAEYEN, Coherent Sheaves over Microstructure Sheaves, UIA preprint (1991), Proceedings of the Colmar meeting.

[RVO2] A. RADWAN AND F. VAN OYSTAEYEN, Micro-structure Sheaves and Quantum Sections over Formal Schemes, UIA preprint (1991), to appear in Bull. Soc. Math. Belg. (1993).

[SVO] R. Sallam and F. Van Oystakyen, A Micro-structure sheaf and Quantum Section over a Projective Scheme, UIA preprint (1990), J. of Algebra (1992).

[Spr] T. Springer, Algebraic Microlocalization, in "Sém. M. P. Malliavin," Lecture Notes in Math., Springer Verlag, Berlin, 1984.

[Schap] P. SchapIRA, "Microdifferential Systems in the Complex Plane," Grundlehren der Math. Wiss. 269, Springer Verlag, Berlin, 1985. 
[SS] S. P. SMith AND J. T. STAFford, Regularity of the Four Dimensional Sklyanin Algebra, Preprint Ann. Arbor (1990).

[Gin] V. GinsBurG, Characteristic Varieties and Vanishing Cycles, Invent. Math. 84 (1986), 327-402.

[WIT] E. WrTten, "Gauge Theories, Vertex Models and Quantum Groups."

[WOR] S. L. Woronowicz, Twisted SU(2)-Groups, an Example of a non-commutative Differential Calculus, Publ. R.I.M.S, Kyoto University 23 (1987), 117-181.

\author{
University of Antwerp \\ UIA \\ Department of Mathematics \\ 2610 Wilrijk \\ BELGIUM
}

Rebut el 14 d'Octubre de 1991 